\title{
Topology of helical fluid flow
}

\author{
Andersen, Morten; Brøns, Morten
}

Published in:

European Journal of Applied Mathematics

DOI:

10.1017/S0956792514000084

\section{Publication date:}

2014

\section{Document Version}

Peer reviewed version

Citation for published version (APA):

Andersen, M., \& Brøns, M. (2014). Topology of helical fluid flow. European Journal of Applied Mathematics, 25(03), 375-396. https://doi.org/10.1017/S0956792514000084

\section{General rights}

Copyright and moral rights for the publications made accessible in the public portal are retained by the authors and/or other copyright owners and it is a condition of accessing publications that users recognise and abide by the legal requirements associated with these rights.

- Users may download and print one copy of any publication from the public portal for the purpose of private study or research.

- You may not further distribute the material or use it for any profit-making activity or commercial gain.

- You may freely distribute the URL identifying the publication in the public portal.

\section{Take down policy}

If you believe that this document breaches copyright please contact rucforsk@kb.dk providing details, and we will remove access to the work immediately and investigate your claim. 


\title{
Topology of helical fluid flow
}

\author{
MORTEN ANDERSEN ${ }^{1}$, MORTEN BRØNS ${ }^{1} \dagger$ \\ ${ }^{1}$ Department of Applied Mathematics and Computer Science and Fluid.DTU, Technical University of \\ Denmark, Building 303B, DK-2800 Kongens Lyngby, Denmark
}

(Received To be entered by editorial office)

Considering a coordinate-free formulation of helical symmetry rather than more traditional definitions based on coordinates, we discuss basic properties of helical vector fields and compare results from the literature obtained with other approaches. In particular, we discuss the role of the stream function for the topology of the streamline pattern in incompressible flows. On this basis, we perform a comprehensive study of the topology of the flow field generated by a helical vortex filament in an ideal fluid. The classical expression for the streamfunction obtained by Hardin (Phys. Fluids 25, 1982, pp. 1949-1952) contains an infinite sum of modified Bessel functions. Using the approach by Okulov (Russ. J. Eng. Thermophys. 5, 1995, 63-75) we obtain a closed-form approximation which is considerably easier to analyse. Critical points of the stream function can be found from the zeroes of a single real function of one variable, and we show that three different flow topologies can occur, depending on a single dimensionless parameter. Including the self-induced velocity on the vortex filament by the localised induction approximation the stream function is slightly modified and an extra parameter is introduced. In this setting two new flow $\dagger$ Email address for correspondence: mobr@dtu.dk 
topologies arise, but not more than two critical points occur for any combination of the parameters.

Key Words: Helical flow, flow topology, vortex filaments, asymptotic expansions.

\section{Introduction}

Helical flows (flows with helical symmetry) arise behind propellers and wind turbines $[19,26]$ and helical vortices are important for helicopter rotor performance $[18,36,30]$. Helical pipe flow has a general engineering interest $[11,24,21,38,17]$ and has also been studied as a model for a blood vessel with non-vanishing curvature and torsion [39, 40].

Helical symmetry combines translational and rotational symmetries and have these as limiting cases. In general, however, flows with helical symmetry are more complicated than what can occur in each of these cases separately. Often helical symmetry is described from a certain coordinate relation $[20,39,16,35,37,9]$. In contrast to this, we will use a coordinate-free approach from which the basic properties of fields with helical symmetry can be obtained in a simple and transparent way. This approach was first used by Ettinger \& Titi [15] to investigate global uniqueness and existence of solutions for inviscid, helical flow. Using the coordinate free approach we give a short proof that the velocity field generated by a helical vortex filament is helical and we show consistency to helical formulations that rely on coordinates.

We then focus on the topological structure of helical flows, and, in particular, bifurcations in the streamline patterns. A topological approach has been used to characterize 
two-dimensional flows $[5,6,13]$ and axisymmetric flows $[8,7]$. For an incompressible helical flow a stream function can be defined, and we discuss to which extent the topology of the streamlines can be inferred from this. As a specific case, we consider the flow induced by a helical vortex filament. At a given helix, the vorticity is tangent to the helix and elsewhere the flow is potential. Hardin [19] found an expression for the stream function in terms of infinite series of products of modified Bessel functions. Okulov [28] and Fukumoto \& Okulov [16] have obtained an efficient closed form approximation to such series and we use this to obtain a topological classification of the flow with the pitch of the helix as a bifurcation parameter.

The flow induced by a vortex filament impacts the location of the vortex filament itself. This can be taken into account using the localised induction approximation (LIA). When LIA is used $[10,33,34]$ a helical filament has the interesting property that the self-induced motion does not spoil the helical shape. However, the filament translates and rotates as shown by e.g. Batchelor [4]. LIA results in a minor modification of the stream function discussed above where the speed of the helix appears as an extra parameter which was investigated by Mezic et al. [27]. We construct a closed form approximation and make a topological analysis of the flow field in this case as well.

This article is organised as follows. In $\S 2$ the notions of helical vector fields and helical scalar functions are introduced as well as the helical vortex filament. Helical coordinates are then described. The stream function and the geometric implications of its topology are discussed. In $\S 3$ the stream function calculated by Hardin, $\psi_{H}$, is introduced. We find a closed form approximation of the stream function, $\psi_{H, c}$, and analyse the topology of 
the induced flow. The velocity field generated by a translating and rotating helical vortex filament is approximated and analysed in $\S 4$. In $\S 5$ conclusions are drawn.

\section{Helical vector fields and scalar functions}

Let $\boldsymbol{e}_{3}$ be a unit vector in $\mathbb{R}^{3}$ and let $R_{\rho}$ denote the counter-clockwise rotation by the angle $\rho$ around $\boldsymbol{e}_{3}$. For a fixed $d \in \mathbb{R}$ we define a family of helical transformations with respect to $\boldsymbol{e}_{3}$ by

$$
S_{\rho} \boldsymbol{x} \equiv R_{\rho} \boldsymbol{x}+\rho d \boldsymbol{e}_{3}
$$

For a fixed $\boldsymbol{x}$, the curve $\rho \rightarrow S_{\rho} \boldsymbol{x}$ is a helix with pitch $2 \pi d$. In a right-handed cartesian coordinate system $\boldsymbol{e}_{1}, \boldsymbol{e}_{2}, \boldsymbol{e}_{3}$, which we will use from now on, we have

$$
R_{\rho} \boldsymbol{x}=\left(\begin{array}{ccc}
\cos (\rho) & -\sin (\rho) & 0 \\
\sin (\rho) & \cos (\rho) & 0 \\
0 & 0 & 1
\end{array}\right) \boldsymbol{x} .
$$

A helical transformation is distance preserving. For a fixed pitch, $2 \pi d, \mathbb{R}^{3}$ may be filled by disjoint helices. All results in the rest of the article rely on the assumption that the pitch is arbitrary but fixed once chosen. Also the axis of rotation is always the $z$-axis. Following Ettinger \& Titi [15] we make two basic definitions

Definition 2.1 A scalar function $f: \mathbb{R}^{3} \mapsto \mathbb{R}$ is called helical if

$$
f\left(S_{\rho} \boldsymbol{x}\right)=f(\boldsymbol{x}), \forall \rho \in \mathbb{R}, \forall \boldsymbol{x} \in \mathbb{R}^{3}
$$

Thus, the value of a helical scalar function is constant along any helix. 
Definition 2.2 The vector field $v: \mathbb{R}^{3} \mapsto \mathbb{R}^{3}$ is called helical if

$$
\boldsymbol{v}\left(S_{\rho} \boldsymbol{x}\right)=R_{\rho} \boldsymbol{v}(\boldsymbol{x}), \forall \rho \in \mathbb{R}, \forall \boldsymbol{x} \in \mathbb{R}^{3} .
$$

Both helical functions and helical vector fields are completely determined by their values in the $x y$-plane: If $\boldsymbol{x}=(x, y, z)$ and $\rho^{\prime}=-z / d$, the point $S_{\rho^{\prime}}(\boldsymbol{x})$ lies in the $x y$-plane and we find from (2.3) and (2.4)

$$
f(\boldsymbol{x})=f\left(S_{\rho^{\prime}} \boldsymbol{x}\right), \quad \boldsymbol{v}(\boldsymbol{x})=R_{-\rho^{\prime}} \boldsymbol{v}\left(S_{\rho^{\prime}} \boldsymbol{x}\right)
$$

\subsection{Properties of helical scalar functions and helical vector fields}

In the theorem below we collect a number of basic properties fulfilled by helical functions and vector fields. We omit the proofs, as the results follow from simple manipulations with definition 2.1 and definition 2.2 .

Theorem 1 (1) If $f$ is a nonzero helical scalar function then $1 / f$ is a helical scalar function.

(2) The product of helical scalar functions is a helical scalar function.

(3) A linear combination of helical scalar functions is a helical scalar function.

(4) For $i \in\{1, \ldots, n\}$ let $f_{i}(\boldsymbol{x})$ be a helical scalar function and $\boldsymbol{v}_{i}(\boldsymbol{x})$ be a helical vector field. Then $\boldsymbol{w}(\boldsymbol{x})=\sum_{i=1}^{n} f_{i}(\boldsymbol{x}) \boldsymbol{v}_{i}(\boldsymbol{x})$ is a helical vector field.

(5) The dot product of helical vector fields is a helical scalar function.

(6) Let $\boldsymbol{u}(\boldsymbol{x})=\sum_{i=1}^{3} f_{j}(\boldsymbol{x}) \boldsymbol{v}_{j}(\boldsymbol{x})$ where the $\boldsymbol{v}_{j}(\boldsymbol{x})$ are helical vector fields constituting 
a basis for $\mathbb{R}^{3}$. Then $\boldsymbol{u}(\boldsymbol{x})$ is a helical vector field if and only if the $f_{j}(\boldsymbol{x})$ are helical scalar functions.

(7) The cross product of two helical vector fields is a helical vector field.

(8) The curl of a helical vector field is a helical vector field.

(9) The gradient of a helical scalar function is a helical vector field.

Property (6) shows a vector field $\boldsymbol{u}$ is helical if and only if the coordinates of $\boldsymbol{u}$ in any helical basis are helical scalar functions. Examples of helical vector fields are $\boldsymbol{e}_{r}, \boldsymbol{e}_{\theta}, \boldsymbol{e}_{z}$, and the normalised tangent to helices $\boldsymbol{t}=\left(1+\frac{r^{2}}{d^{2}}\right)^{-1 / 2}\left(\boldsymbol{e}_{z}+\frac{r}{d} \boldsymbol{e}_{\theta}\right)$ whereas $\boldsymbol{e}_{1}$ and $\boldsymbol{e}_{2}$ are not helical.

\subsection{The helical vortex filament}

To illustrate the use of the coordinate free description we turn to a specific case - the flow induced by a helical vortex filament. In an inviscid fluid a helical filament is placed with vorticity pointing along the tangent of the helix. The vorticity is zero except at the filament. Assuming the velocity goes to zero at infinite distance the velocity field $\boldsymbol{u}$ can be calculated from the Biot-Savart law. Using the coordinate free form we give a simple proof that the velocity field generated by a number of helical vortex filaments is helical.

The vector potential $\boldsymbol{A}$ satisfies the relation

$$
\boldsymbol{u}=\nabla \times \boldsymbol{A} .
$$


For any vortex filament $c$ in an inviscid fluid $\boldsymbol{A}$ can be found from

$$
\boldsymbol{A}=\frac{\Gamma}{4 \pi} \int_{c} \frac{1}{\|\Delta \boldsymbol{r}\|} d \boldsymbol{l}
$$

see e.g. [3] where $d \boldsymbol{l}$ is the tangent to the filament and $\Delta \boldsymbol{r}$ is the distance from $\boldsymbol{x}$ to a point on the filament which is parametrised by $l$. A helical filament can be parametrised as $S_{l}(\boldsymbol{y})$ for a fixed $\boldsymbol{y}$ and since $d \boldsymbol{l}=\boldsymbol{t}\left(S_{l} \boldsymbol{y}\right) d l=R_{l} \boldsymbol{t}(y) d l$ we have

$$
\boldsymbol{A}(\boldsymbol{x})=\frac{\Gamma}{4 \pi} \int_{-\infty}^{\infty} \frac{1}{\left\|\boldsymbol{x}-S_{l} \boldsymbol{y}\right\|} \boldsymbol{t}\left(S_{l} \boldsymbol{y}\right) d l=\frac{\Gamma}{4 \pi} \int_{-\infty}^{\infty} \frac{1}{\left\|\boldsymbol{x}-S_{l} \boldsymbol{y}\right\|} R_{l} \boldsymbol{t}(\boldsymbol{y}) d l
$$

Lemma 1 The vector potential $\boldsymbol{A}$ given by equation (2.8) is helical.

Proof The proof is by direct use of definition 2.2. Fix any $\rho \in \mathbb{R}$ :

$$
R_{\rho} \boldsymbol{A}(\boldsymbol{x})=\frac{\Gamma}{4 \pi} R_{\rho} \int_{-\infty}^{\infty} \frac{1}{\left\|\boldsymbol{x}-S_{l} \boldsymbol{y}\right\|} R_{l} \boldsymbol{t}(\boldsymbol{y}) d l=\frac{\Gamma}{4 \pi} \int_{-\infty}^{\infty} \frac{1}{\left\|\boldsymbol{x}-S_{l} \boldsymbol{y}\right\|} R_{\rho+l} \boldsymbol{t}(\boldsymbol{y}) d l
$$

This is now compared to:

$$
\boldsymbol{A}\left(S_{\rho} \boldsymbol{x}\right)=\frac{\Gamma}{4 \pi} \int_{-\infty}^{\infty} \frac{1}{\left\|S_{\rho} \boldsymbol{x}-S_{l} \boldsymbol{y}\right\|} R_{l} \boldsymbol{t}(\boldsymbol{y}) d l
$$

Changing the integration variable $l=\rho+s$ in equation (2.10) yields

$$
\boldsymbol{A}\left(S_{\rho} \boldsymbol{x}\right)=\frac{\Gamma}{4 \pi} \int_{-\infty}^{\infty} \frac{1}{\left\|S_{\rho} \boldsymbol{x}-S_{\rho+s} \boldsymbol{y}\right\|} R_{\rho+s} \boldsymbol{t}(\boldsymbol{y}) d s
$$

Since $S_{\rho+s} \boldsymbol{y}=S_{\rho}\left(S_{s} \boldsymbol{y}\right)$ we get using that a helical transformation conserves distance i.e. $|\boldsymbol{y}-\boldsymbol{x}|=\left|S_{\rho} \boldsymbol{y}-S_{\rho} \boldsymbol{x}\right|:$

$$
\boldsymbol{A}\left(S_{\rho} \boldsymbol{x}\right)=\frac{\Gamma}{4 \pi} \int_{-\infty}^{\infty} \frac{1}{\left\|\boldsymbol{x}-S_{s} \boldsymbol{y}\right\|} R_{\rho+s} \boldsymbol{t}(\boldsymbol{y}) d s
$$

which is identical to equation (2.9). 
The argument generalises to multiple helical vortex filaments with the same pitch since equation (2.7) is then a sum of helical terms. Furthermore, since the curl of a helical vector field is helical by property (8) of theorem 1 it follows that $\boldsymbol{u}$ in equation (2.6) is helical.

\subsection{Helical coordinates}

Consider cylindrical coordinates $(r, \theta, z)$ and define the corresponding helical coordinates $\boldsymbol{\xi}=(\xi, \eta, \zeta)$ through the following relations:

$$
\begin{aligned}
& \xi=r \\
& \eta=\theta-\frac{1}{d} z \\
& \zeta=\frac{1}{d} z
\end{aligned}
$$

Since cylindrical coordinates are not well defined on the $z$-axis neither are helical coordinates. Helical coordinates are convenient since the radius $\xi$ and the 'helical angle' $\eta$ are constant on a helix.

Moving on a helix corresponds to changing only $\zeta$. A helix is a straight line parallel to the $\zeta$ axis in the $(\xi, \eta, \zeta)$ coordinate system. Since a helical scalar function is invariant along a helix, this means a scalar function is helical if and only if it is independent of $\zeta$. Hence we have

Lemma 2 A scalar function $f: \mathbb{R}^{3} \mapsto \mathbb{R}$ is helical if and only if $\frac{\partial f(\xi, \eta, \zeta)}{\partial \zeta}=0$. 
A vector field is helical if and only if the coordinates in a helical basis are helical scalar functions according to property $(6)$ :

Lemma 3 A vector field is helical if and only if the coordinates in a helical basis differentiated with respect to $\zeta$ vanishes.

Since $\boldsymbol{e}_{r}, \boldsymbol{e}_{\theta}$ and $\boldsymbol{e}_{z}$ constitute a helical basis a vector field expressed in cylindrical coordinates $\left(u_{r}, u_{\theta}, u_{z}\right)$, is helical if and only if $\frac{\partial}{\partial \zeta} u_{r}=\frac{\partial}{\partial \zeta} u_{\theta}=\frac{\partial}{\partial \zeta} u_{z}=0$.

Finally we have

Lemma 4 If $f$ is a smooth helical scalar function then $\frac{\partial^{i} \partial^{j} \partial^{k}}{\partial \xi^{i} \partial \eta^{j} \partial \zeta^{k}} f$ is a helical scalar function.

Lemma 4 is proved using lemma 2 and that taking derivatives commutes.

Fixing two of three helical coordinates and varying the third, a curve in $\mathbb{R}^{3}$ is generated. The tangent to such a curve, normalised to unity, is the corresponding unit vector to the coordinate direction. We find

$$
e_{\xi}=e_{r}, \quad e_{\eta}=e_{\theta}, \quad e_{\zeta}=t
$$

$\boldsymbol{e}_{\xi}, \boldsymbol{e}_{\eta}, \boldsymbol{e}_{\zeta}$ are helical vector fields and they constitute a basis for $\mathbb{R}^{3}$ except at the $z$ axis. Notice that for points in the $x y$-plane $\zeta=0$ and $(\xi, \eta)=(r, \theta)$ are standard polar coordinates. 


\subsection{Stream function}

Assuming the velocity field is incompressible, $\nabla \cdot \boldsymbol{u}=0$, a stream function can be constructed. In cylindrical coordinates the incompressibility condition is

$$
\frac{1}{r} \frac{\partial}{\partial r}\left(r u_{r}\right)+\frac{1}{r} \frac{\partial u_{\theta}}{\partial \theta}+\frac{\partial u_{z}}{\partial z}=0
$$

The velocity field in cylindrical coordinates can be represented by the velocity field in helical coordinates

$$
u_{r}=u_{\xi}, \quad u_{\theta}=u_{\eta}+\frac{\xi}{\sqrt{d^{2}+\xi^{2}}} u_{\zeta}, \quad u_{z}=\frac{d}{\sqrt{d^{2}+\xi^{2}}} u_{\zeta}
$$

and inserted in equation (2.15)

$$
\frac{1}{\xi} \frac{\partial}{\partial \xi}\left(\xi u_{\xi}\right)+\frac{1}{\xi} \frac{\partial}{\partial \theta}\left(u_{\eta}+\frac{\xi}{\sqrt{d^{2}+\xi^{2}}} u_{\zeta}\right)+\frac{\partial}{\partial z}\left(\frac{d}{\sqrt{d^{2}+\xi^{2}}} u_{\zeta}\right)=0
$$

From equation (2.13) the chain rule yields

$$
\frac{\partial}{\partial r}=\frac{\partial}{\partial \xi}, \quad \frac{\partial}{\partial \theta}=\frac{\partial}{\partial \eta}, \quad \frac{\partial}{\partial z}=\frac{1}{d}\left(-\frac{\partial}{\partial \eta}+\frac{\partial}{\partial \zeta}\right)
$$

from which we can transform equation (2.17)

$$
\frac{1}{\xi}\left(\frac{\partial}{\partial \xi}\left(\xi u_{\xi}\right)+\frac{\partial}{\partial \eta} u_{\eta}\right)+\frac{1}{\sqrt{d^{2}+\xi^{2}}} \frac{\partial}{\partial \zeta} u_{\zeta}=0
$$

For a helical vector field the last term vanishes by lemma 3. Then equation (2.19) yields

$$
\frac{\partial\left(\xi u_{\xi}\right)}{\partial \xi}+\frac{\partial u_{\eta}}{\partial \eta}=0
$$

from which it follows that a stream function $\psi: \mathbb{R}^{2} \rightarrow \mathbb{R}$ exists such that

$$
\frac{\partial \psi}{\partial \eta}=\xi u_{\xi}, \quad-\frac{\partial \psi}{\partial \xi}=u_{\eta}
$$

It follows that $u_{\xi} \boldsymbol{e}_{\xi}+u_{\eta} \boldsymbol{e}_{\eta}$ is parallel to the tangent of the level curves of the stream function. 
A helical, incompressible velocity field thus only depends on two single valued functions $\psi(\xi, \eta)$ and $u_{\zeta}(\xi, \eta)$ and can always be written

$$
\boldsymbol{u}=\frac{1}{\xi} \frac{\partial \psi(\xi, \eta)}{\partial \eta} \boldsymbol{e}_{r}-\frac{\partial \psi(\xi, \eta)}{\partial \xi} \boldsymbol{e}_{\theta}+u_{\zeta}(\xi, \eta) \boldsymbol{t}
$$

If the velocity is known in the $x y$-plane it is easy to calculate the entire velocity field from equation $(2.5)$.

As $d \rightarrow \infty, t \rightarrow \boldsymbol{e}_{z}$ and $\eta \rightarrow \theta$, and the flow will have translational symmetry along the $\boldsymbol{e}_{3}$-axis. This agrees with equation (2.22) where we recover the standard stream function formulation in cylindrical coordinates for flows with translational symmetry. If further $u_{\zeta}=u_{z}=0$, the flow is two-dimensional. It is common to use a stream function depending on $\xi$ and $\eta$ spanned in the plane normal to the tangent vector $[16,26,2,39$, 23, 12]. References are often to the work of Landman [22] and Dritschel [14] where a helical, incompressible velocity field is decomposed in the form

$$
\boldsymbol{u}=\nabla f_{1} \times \boldsymbol{B}+f_{2} \boldsymbol{B}
$$

where $\boldsymbol{B}$ is the tangent to the helices not normalised to unity but to be a Beltrami vector

$$
\boldsymbol{B}=\left(1+\frac{r^{2}}{d^{2}}\right)^{-1}\left(\boldsymbol{e}_{z}+\frac{r}{d} \boldsymbol{e}_{\theta}\right)
$$

Landman [22] requires $f_{1}$ and $f_{2}$ are helical scalar functions i.e. depend on $\xi, \eta$ (and time). Dritschel [14] does not explicitly require $f_{1}$ and $f_{2}$ being helical. Here $f_{1}$ plays the role of the stream function. From theorem 1 it is clear that $\boldsymbol{u}$ in equation (2.23) is helical for helical $f_{1}$ and $f_{2}$. However, we have found no proof in literature why all helical velocity field can be written in the form of equation (2.23). Actually, Childress et al. 
[9] define a helical velocity field to be one that obeys equation (2.23). This definition is equivalent to our definition 2.2 :

Lemma 5 An incompressible vector field, $\boldsymbol{u}$, is helical if and only if there exists helical scalar functions $f_{1}$ and $f_{2}$ such that equation (2.23) holds.

Proof The proof relies on that any helical, incompressible vector field can be written in the form of equation (2.22). First assume $f_{1}$ and $f_{2}$ are helical scalar functions. Using the gradient for cylindrical coordinates and equation (2.18), equation (2.14) and

$$
\boldsymbol{t}=\frac{1}{\sqrt{1+\frac{r^{2}}{d^{2}}}}\left(\boldsymbol{e}_{z}+\frac{r}{d} \boldsymbol{e}_{\theta}\right)
$$

then equation (2.23) can be written as

$$
\boldsymbol{u}=\frac{1}{\xi} \frac{\partial f_{1}}{\partial \eta} \boldsymbol{e}_{r}-\frac{\partial f_{1}}{\partial \xi} \boldsymbol{e}_{\eta}+\frac{1}{\sqrt{1+\frac{\xi^{2}}{d^{2}}}}\left(\frac{\xi}{d} \frac{\partial f_{1}}{\partial \xi}+f_{2}\right) \boldsymbol{t}
$$

Choosing

$$
\psi=f_{1}, \quad u_{\zeta}=\frac{1}{\sqrt{1+\frac{\xi^{2}}{d^{2}}}}\left(\frac{\xi}{d} \frac{\partial f_{1}}{\partial \xi}+f_{2}\right),
$$

equation (2.22) is recovered. To prove the converse statement assume $\psi$ and $u_{\zeta}$ are helical scalar functions. Invert equation (2.27) to define $f_{1}$ and $f_{2}$ which are helical scalar functions by lemma 4 and theorem 1 . Then we get equation (2.26) which is equivalent to equation (2.23). 


\subsection{Interpretation of level curves of $\psi$}

Figure 1(a) shows an example of a contour plot of $\psi$ in the $x y$ plane. Three critical points are shown, two elliptic and one hyperbolic. A critical point of $\psi$ is characterised by $\frac{\partial}{\partial \xi} \psi(\xi, \eta)=\frac{\partial}{\partial \eta} \psi(\xi, \eta)=0$ i.e. $u_{\xi}=u_{\eta}=0$ so in the three dimensional flow only $u_{\zeta}$ may be nonzero. The velocity field at some height $z=z_{1}$ is merely a rotation around the $z$-axis of the velocity field at $z=0$. It is appreciable that the stream function lives in the $x y$-plane since we just have to rotate this around the $z$-axis as well. This is illustrated in figure 1.

A critical point of $\psi$ in a plane with constant $z$-value corresponds to flow tangential to a helix in the three dimensional flow. Since a helical flow does not depend on $\zeta$ the flow has constant magnitude along any helix including this special 'critical point helix'. This is illustrated for the middle critical point in figure 1 . The tangent to a contour of $\psi$ gives two velocity coordinates according to the properties of the stream function. In two dimensions (figure 1(a)) a closed curve of $\psi$ separates the flow. The same is true in three dimensions corresponding to a helix tube that separates the flow. This is shown in figure 1 for the closed curve surrounding the middle critical point in two dimensions. Thus, a critical point of $\psi$ corresponds to a stream line in the three dimensional flow and a level curve of $\psi$ corresponds to a stream surface of the three dimensional flow.

Through each point in the $x y$ - plane passes exactly one helix. On each of these helices $u_{\zeta}$ is constant. However, $u_{\zeta}$ may change for different points in the $x y$ - plane. The helical tube in figure 1 thus may have different $u_{\zeta}$ for different helices. The value of $u_{\zeta}$ can not be deduced from the stream function in general. 
(a)

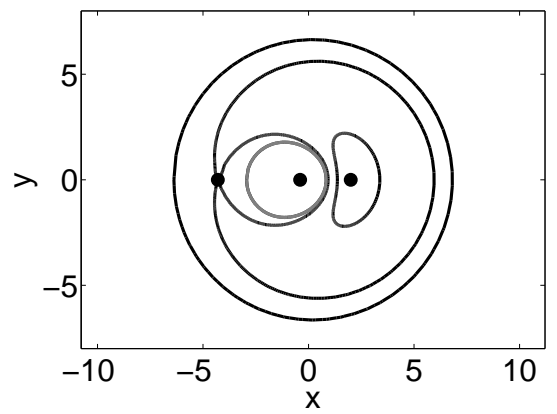

(b)

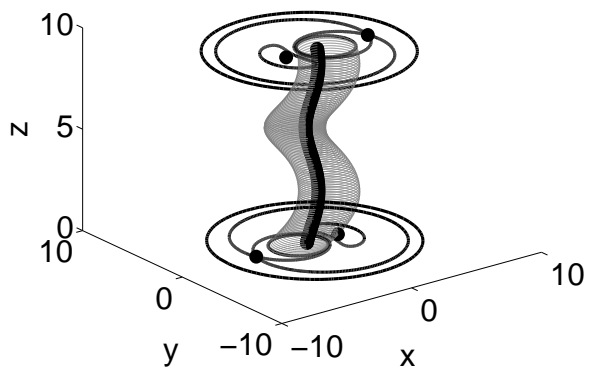

Figure 1. (a) Example of contourplot of the stream function. The tangent to the contour curves of $\psi$ gives two of the velocity coordinates. (b) The corresponding three dimensional picture. A critical point in (a) corresponds to a helix in (b) with a constant velocity vector parallel to the tangent of the helix. A closed curve in (a) corresponds to a helix tube in (b) with no cross flow.

There is an important difference between the interpretation of level curves of the stream function in helical symmetry and in translation symmetry relating to the vorticity $\boldsymbol{\omega}=\nabla \times \boldsymbol{u}$. From Kelvin's circulation theorem we have

$$
\Gamma=\int_{C} \boldsymbol{u} \cdot \boldsymbol{d l}=\int_{S} \boldsymbol{\omega} \cdot \boldsymbol{n} d s
$$

with $C$ being a closed curve with tangent $\boldsymbol{d} \boldsymbol{l}$ and surface $S$ with normal $\boldsymbol{n}$. For a smooth vorticity distribution and a small surface with area $S$ this yields for the the normal component of the vorticity $\omega_{\perp}$

$$
\Gamma \approx \omega_{\perp} S
$$

Therefore, nonzero $\Gamma$ means nonzero vorticity. Now, let $C$ be a small closed level curve of $\psi$ encircling a critical point. Such curves can be found in the case shown in figure 1(a). First imagine this is a translational symmetric case which implies the third basis vector is $\boldsymbol{e}_{3}$. Then the value of $u_{3}$ has no impact on $\boldsymbol{u} \cdot \boldsymbol{d} \boldsymbol{l}$. The $x y$ - component of the flow 
is parallel to the tangent to the level curve of $\psi$. This means $\boldsymbol{u} \cdot \boldsymbol{d l}$ is nonzero and has constant sign for any point on the curve. Therefore $\Gamma=\int_{C} \boldsymbol{u} \cdot \boldsymbol{d l}$ is nonzero. Thus, in the translation symmetric case the vorticity is non-zero at an elliptic critical point.

In a helical flow using $\boldsymbol{e}_{r}, \boldsymbol{e}_{\theta}, \boldsymbol{t}$ as a basis the situation is different since the third basis vector $\boldsymbol{t}$ is not orthogonal to the $x y$ - plane. Now $\boldsymbol{u} \cdot \boldsymbol{d} \boldsymbol{l}$ also depends on $u_{\zeta}$. Therefore, a closed level curve of $\psi$ may have zero circulation and the vorticity at an elliptic critical point of $\psi$ may be zero.

The character of a critical point can be determined by the second derivative test

$$
H=\frac{1}{\xi^{2}}\left(\frac{\partial^{2} \psi}{\partial \xi^{2}} \frac{\partial^{2} \psi}{\partial \eta^{2}}-\left(\frac{\partial^{2} \psi}{\partial \xi \partial \eta}\right)^{2}\right)
$$

where the right side is evaluated at the critical point. If $H>0$ the critical point is elliptic. If $H<0$ the critical point is hyperbolic.

\section{The velocity field generated by a helical vortex filament}

Hardin [19] found the stream function for the flow generated by the vortex filament of strength $\Gamma$, radius $a$ and pitch $d$ as an infinite series involving modified Bessel functions,

$$
\psi_{H}=\frac{\Gamma}{2 \pi}\left(\begin{array}{c}
\frac{\xi^{2}}{2 d^{2}} \\
-\ln (\xi)+\ln (a)+\frac{a^{2}}{2 d^{2}}
\end{array}\right)-\frac{\Gamma a \xi}{\pi d^{2}} \sum_{m=1}^{\infty}\left(\begin{array}{c}
K_{m}^{\prime}\left(m \frac{a}{d}\right) I_{m}^{\prime}\left(m \frac{\xi}{d}\right) \\
K_{m}^{\prime}\left(m \frac{\xi}{d}\right) I_{m}^{\prime}\left(m \frac{a}{d}\right)
\end{array}\right) \cos (m \eta)
$$

Here the top line is valid for $\xi<a$ (the inner solution) and the lower line is valid for $\xi>a$ (the outer solution). We have included a constant $\ln (a)+\frac{a^{2}}{2 d^{2}}$ not in the original expression by Hardin to ensure $\psi_{H}$ is continuous at the helix cylinder $\xi=a$. 
We introduce scaled variables

$$
\tilde{\xi}=\frac{\xi}{d}, \quad \lambda=\frac{a}{d},
$$

such that the helix cylinder is at $\tilde{\xi}=\lambda$, and define $\tilde{\psi}_{H}(\tilde{\xi}, \eta)=\frac{\pi}{\Gamma} \psi_{H}(d \tilde{\xi}, \eta)$ :

$$
\tilde{\psi}_{H}(\tilde{\xi}, \eta)=\frac{1}{2}\left(\begin{array}{c}
\frac{\tilde{\xi}^{2}}{2} \\
-\ln (\tilde{\xi})+\ln (\lambda)+\frac{\lambda^{2}}{2}
\end{array}\right)-\tilde{\xi} \lambda \sum_{m=1}^{\infty}\left(\begin{array}{c}
K_{m}^{\prime}(m \lambda) I_{m}^{\prime}(m \tilde{\xi}) \\
K_{m}^{\prime}(m \tilde{\xi}) I_{m}^{\prime}(m \lambda)
\end{array}\right) \cos (m \eta)
$$

We now skip the tilde for convenience (whenever $\lambda$ is appearing we are using scaled variables). In the rest of the paper we focus on the topology of the flow induced by helical vortex filaments and proceed to state a few basic facts about the critical points of $\psi_{H}$ which can be obtained from equation (3.3).

It is immediately clear that $\eta=0$ and $\eta=\pi$ solve $\frac{\partial}{\partial \eta} \psi_{H}=0$. Whether there are further solutions is not clear, but a numerical investigation by Mezic et al. [27] finds no critical points at other values of $\eta$.

The modified Bessel functions are solutions to the equation

$$
x^{2} y^{\prime \prime}+x y^{\prime}-\left(x^{2}+m^{2}\right) y=0 \text {. }
$$

Using this and the well-known properties $K_{m}(x)>0, I_{m}(x)>0, I_{m}^{\prime}(x)>0, K_{m}^{\prime}(x)<0$ for $x>0$ one finds that $\frac{\partial}{\partial \xi} \psi(\xi, 0)$ has constant sign in the inner and the outer domain. Thus, there are no critical points at $\eta=0$, but they may occur at $\eta=\pi$. The type of a critical point is determined by the Hessian (2.30). It is easily seen that

$$
\frac{\partial^{2}}{\partial \eta \partial \xi} \psi_{H}(\xi, \pi)=0
$$


while the signs of $\frac{\partial^{2}}{\partial \xi^{2}} \psi_{H}(\xi, \pi)$ and $\frac{\partial^{2}}{\partial \eta^{2}} \psi_{H}(\xi, \pi)$ are not obvious.

\subsection{Closed form approximation of $\psi_{H}$}

The infinite sum appearing in $\psi_{H}$ makes analysis hard, but there are also numerical issues due to the asymptotic behaviour of the Bessel functions. While $I_{m}^{\prime}$ goes to infinity for large arguments, $K_{m}^{\prime}$ goes to zero. Hence products of the form $I_{m}^{\prime}(m x) K_{m}^{\prime}(m y)$ may be impossible to evaluate numerically from each of the factors. Mezic et al. [27] use a finite number of terms to represent the infinite series without addressing this issue while Ijzermans et al. [20] use an asymptotic expansion which is only briefly discussed. This asymptotic expansion may be related to the approach by Okulov [28] and Fukumoto \& Okulov [16] we discuss now. In this approach approximations for the modified Bessel functions for large arguments are used, see Abramowitz \& Stegun [1, p. 378] and Olver $[31,32]$ for a derivation. The resulting closed-form approximation to $\psi_{H}$ is most useful, but unfortunately there are some misprints. In the following we rederive the closed-form approximation for equation (3.3).

Define

$$
f(x)=\sqrt{1+x^{2}}+\ln \left(\frac{x}{1+\sqrt{1+x^{2}}}\right) .
$$

For later use we notice that $f$ is strictly increasing for positive $x$. Then for large $m$

$$
\begin{aligned}
I_{m}^{\prime}(m x) & \approx \frac{1}{\sqrt{2 \pi m}} \frac{\left(1+x^{2}\right)^{\frac{1}{4}}}{x} e^{m f(x)} \\
K_{m}^{\prime}(m y) & \approx-\sqrt{\frac{\pi}{2 m}} \frac{\left(1+y^{2}\right)^{\frac{1}{4}}}{y} e^{-m f(y)} .
\end{aligned}
$$


With these we obtain (Re denotes real part)

$$
\begin{aligned}
& \sum_{m=1}^{\infty} K_{m}^{\prime}(m y) I_{m}^{\prime}(m x) \cos (m \eta)=\operatorname{Re}\left(\sum_{m=1}^{\infty} K_{m}^{\prime}(m y) I_{m}^{\prime}(m x) e^{\mathrm{i} m \eta}\right) \\
& \approx-\operatorname{Re}\left(\sum_{m=1}^{\infty} \sqrt{\frac{\pi}{2 m}} \frac{\left(1+y^{2}\right)^{\frac{1}{4}}}{y} e^{-m f(y)} \frac{1}{\sqrt{2 \pi m}} \frac{\left(1+x^{2}\right)^{\frac{1}{4}}}{x} e^{m f(x)} e^{\mathrm{i} m \eta}\right) \\
& =-\frac{\left(\left(1+x^{2}\right)\left(1+y^{2}\right)\right)^{\frac{1}{4}}}{2 x y} \operatorname{Re}\left(\sum_{m=1}^{\infty} \frac{1}{m} e^{-m(f(y)-f(x)-\mathrm{i} \eta)}\right) .
\end{aligned}
$$

This can be further simplified by noting that the well-known formula

$$
\sum_{m=1}^{\infty}(-1)^{m+1} \frac{x^{m}}{m}=\ln (1+x) \quad \text { for }|x|<1
$$

yields

$$
\sum_{m=1}^{\infty} \frac{1}{m} e^{-m(\alpha-\mathrm{i} \beta)}=-\ln \left(1-e^{-\alpha+\mathrm{i} \beta}\right) \text { for } \alpha>0 .
$$

Note that Okulov [29] and Fukumoto \& Okulov [16] have a sign error in this expression. Consider now equation (3.8) with $y>x$. Then $f(y)>f(x)$ so equation (3.10) can be used to get the approximation

$$
\sum_{m=1}^{\infty} K_{m}^{\prime}(m y) I_{m}^{\prime}(m x) \cos (m \eta) \approx \frac{\left(\left(1+x^{2}\right)\left(1+y^{2}\right)\right)^{\frac{1}{4}}}{2 x y} \operatorname{Re}\left(\ln \left(1-e^{f(x)-f(y)+\mathrm{i} \eta}\right)\right) \text {. }
$$

Denoting

$$
\alpha=\alpha(\xi, \eta)=f(\xi)-f(\lambda)
$$

we apply equation (3.11) to equation (3.3) to finally get a closed form approximation (denoted by subscript $c$ ) of Hardin's solution, 
(a)

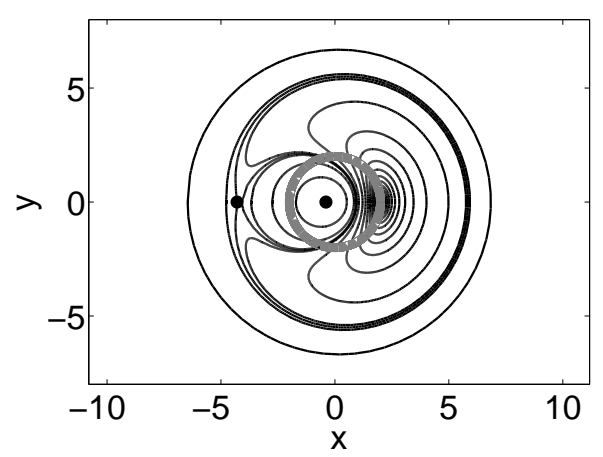

(b)

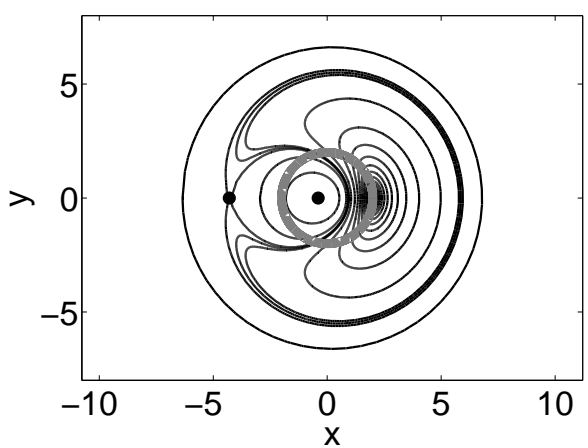

Figure 2. Level curves of the stream function, with the cylinder $r=\lambda=2$ marked as a grey circle. Critical points of $\psi_{H, c}$ are marked with black dots. (a) Level curves of $\psi_{H}$, equation (3.3), including 300 terms (b) Level curves of $\psi_{H, c}$, equation (3.13).

$\psi_{H, c}(\xi, \eta)=\frac{1}{2}\left(\begin{array}{c}\frac{\xi^{2}}{2} \\ -\ln (\xi)+\ln (\lambda)+\frac{\lambda^{2}}{2}\end{array}\right)-\frac{1}{2}\left(\left(1+\lambda^{2}\right)\left(1+\xi^{2}\right)\right)^{\frac{1}{4}} \operatorname{Re}\left(\ln \left(1-e^{ \pm \alpha+\mathrm{i} \eta}\right)\right)$.

The limit of equation (3.13) as $\xi$ tends to $\lambda$ exists for nonzero $\eta$. There only is a problem on the filament itself $(\xi, \eta)=(\lambda, 0)$ where the velocity field diverges similar to the situation in point vortex dynamics.

An typical example of level curves for $\psi_{H}$ and $\psi_{H, c}$ is shown in figure 2. In this example, and in many other we have tested, a very good agreement between the series and the closed form is achieved. 


\subsection{Topological analysis of the closed form expression}

We now analyse the topology of the flow induced by the closed form expression equation (3.13). To find critical points we compute first the partial derivative

$$
\frac{\partial}{\partial \eta} \psi_{H, c}(\xi, \eta)=\frac{1}{2}\left(\left(1+\lambda^{2}\right)\left(1+\xi^{2}\right)\right)^{\frac{1}{4}} \operatorname{Re}\left(\frac{\mathrm{i} e^{ \pm \alpha+\mathrm{i} \eta}}{1-e^{ \pm \alpha+\mathrm{i} \eta}}\right)
$$

which is zero only when $\eta=0, \pi$. These values are also zeroes for the original expression equation (3.3), but for the closed form it is clear that there are no other solutions. The other partial derivative is

$$
\begin{aligned}
& \frac{\partial}{\partial \xi} \psi_{H, c}(\xi, \eta)=\frac{1}{2}\left(\begin{array}{c}
\xi \\
-\frac{1}{\xi}
\end{array}\right) \\
& \quad-\frac{1}{2}\left(1+\lambda^{2}\right)^{\frac{1}{4}}\left(\frac{1}{2} \frac{\xi}{\left(1+\xi^{2}\right)^{\frac{3}{4}}} \operatorname{Re}\left(\ln \left(1-e^{ \pm \alpha+\mathrm{i} \eta}\right)\right) \mp \frac{\left(1+\xi^{2}\right)^{\frac{3}{4}}}{\xi} \operatorname{Re}\left(\frac{e^{ \pm \alpha+\mathrm{i} \eta}}{1-e^{ \pm \alpha+\mathrm{i} \eta}}\right)\right) .
\end{aligned}
$$

Inserting $\eta=0$, we obtain

$$
\begin{aligned}
\frac{\partial}{\partial \xi} \psi_{H, c}(\xi, 0)= & \frac{1}{2}\left(\begin{array}{c}
\xi \\
-\frac{1}{\xi}
\end{array}\right) \\
& -\frac{1}{2}\left(1+\lambda^{2}\right)^{\frac{1}{4}}\left(\frac{1}{2} \frac{\xi}{\left(1+\xi^{2}\right)^{\frac{3}{4}}} \ln \left(1-e^{ \pm \alpha}\right) \mp \frac{\left(1+\xi^{2}\right)^{\frac{3}{4}}}{\xi} \frac{e^{ \pm \alpha}}{1-e^{ \pm \alpha}}\right) .
\end{aligned}
$$

Lemma 6 There are no critical points of $\psi_{H, c}(\xi, 0)$ off the filament. 
Proof We will show that $\frac{\partial}{\partial \xi} \psi_{H, c}(\xi, 0)$ has one sign away from the helix cylinder. It is easy to see that

$$
\frac{\partial}{\partial \xi} \psi_{H, c}(\xi, 0)>0 \text { for } \xi<\lambda
$$

since all three terms in equation (3.16) are positive.

To show that $\frac{\partial}{\partial \xi} \psi_{H, c}(\xi, 0) \neq 0$ also for $\xi>\lambda$ we go back to equation (3.13). We see that $\frac{\partial}{\partial \xi} \psi_{H, c}(\xi, 0)<0$ if $\frac{\partial}{\partial \xi}\left(-\left(1+\xi^{2}\right)^{\frac{1}{4}} \operatorname{Re}\left(\ln \left(1-e^{-\alpha}\right)\right)\right)<0$. From equation (3.10) we get

$$
-\left(1+\xi^{2}\right)^{\frac{1}{4}} \operatorname{Re}\left(\ln \left(1-e^{-\alpha}\right)\right)=\left(1+\xi^{2}\right)^{\frac{1}{4}} \sum_{m=1}^{\infty} \frac{1}{m} e^{-m \alpha} .
$$

Then

$$
\begin{aligned}
\frac{\partial}{\partial \xi}\left(-\left(1+\xi^{2}\right)^{\frac{1}{4}} \operatorname{Re}\left(\ln \left(1-e^{-\alpha}\right)\right)\right) & \\
& \sum_{m=1}^{\infty} e^{-m \alpha}\left(\frac{1}{2 m} \frac{\xi}{\left(1+\xi^{2}\right)^{\frac{3}{4}}}-\frac{\left(1+\xi^{2}\right)^{\frac{3}{4}}}{\xi}\right) .
\end{aligned}
$$

It is not difficult to see that each term in equation (3.19) is negative for $\xi>0$ such that

$$
\frac{\partial}{\partial \xi} \psi_{H, c}(\xi, 0)<0 \text { for } \xi>\lambda
$$

so combining (3.17) and (3.20) we see that no critical points exist at $\eta=0$.

We now turn to $\eta=\pi$ and introduce

$$
\begin{aligned}
F(\xi, \lambda) \equiv \frac{\partial}{\partial \xi} \psi_{H, c}(\xi, \pi) & =\frac{1}{2}\left(\begin{array}{c}
\xi \\
-\frac{1}{\xi}
\end{array}\right) \\
& -\frac{1}{2}\left(1+\lambda^{2}\right)^{\frac{1}{4}}\left(\frac{1}{2} \frac{\xi}{\left(1+\xi^{2}\right)^{\frac{3}{4}}} \ln \left(1+e^{ \pm \alpha}\right) \pm \frac{\left(1+\xi^{2}\right)^{\frac{3}{4}}}{\xi} \frac{e^{ \pm \alpha}}{1+e^{ \pm \alpha}}\right),
\end{aligned}
$$


the zeroes of which represent critical points. To determine the type of a possible critical point we find by direct computation

$$
\frac{\partial^{2} \psi_{H, c}(\xi, \pi)}{\partial \xi \partial \eta}=\frac{\partial F(\xi, \lambda)}{\partial \eta}=0, \frac{\partial^{2} \psi_{H, c}(\xi, \pi)}{\partial \eta^{2}}>0, \text { for } \xi \neq \lambda
$$

such that the sign of the Hessian (2.30) is solely determined by the sign of $\frac{\partial^{2} \psi_{H, c}(\xi, \pi)}{\partial \xi^{2}}=$ $\frac{\partial F(\xi, \lambda)}{\partial \xi}$. If it is positive the critical point is a center and if it is negative it is a saddle. Hence, the function $F$ contains all information about existence and properties of critical points.

Lemma $7 F(\xi, \lambda)$ is negative for fixed $\lambda$ and $\xi$ small or $\xi$ large.

Proof We consider first small $\xi$. Using L'Hospital's rule on

$$
\xi \ln \left(1+e^{\alpha}\right)=\frac{\ln \left(1+e^{f(\xi)-f(\lambda)}\right)}{\frac{1}{\xi}}
$$

which appears in equation (3.21) we get

$$
\lim _{\xi \rightarrow 0} \xi \ln \left(1+e^{\alpha}\right)=\lim _{\xi \rightarrow 0}-\xi \sqrt{1+\xi^{2}} \frac{e^{f(\xi)-f(\lambda)}}{1+e^{f(\xi)-f(\lambda)}}=0 .
$$

The limits of the rest of the terms in equation (3.21) are easily obtained, yielding

$$
\lim _{\xi \rightarrow 0} F(\xi, \lambda)=-\frac{1}{4}\left(1+\lambda^{2}\right)^{\frac{1}{4}} e^{1-f(\lambda)}<0 .
$$

For large $\xi$ we also have $F(\lambda, \xi)<0$ : In the outer region we have, by disregarding a negative term,

$$
\begin{aligned}
F(\xi, \lambda) & <-\frac{1}{2 \xi}+\frac{1}{2}\left(1+\lambda^{2}\right)^{\frac{1}{4}} \frac{\left(1+\xi^{2}\right)^{\frac{3}{4}}}{\xi} \frac{e^{-\alpha}}{1+e^{-\alpha}} \\
& =\frac{-\left(e^{\alpha}+1\right)+\left(1+\lambda^{2}\right)^{\frac{1}{4}}\left(1+\xi^{2}\right)^{\frac{3}{4}}}{2 \xi\left(e^{\alpha}+1\right)} .
\end{aligned}
$$


As $\xi>\lambda$ it further follows that

$$
F(\xi, \lambda)<\frac{-\left(e^{\alpha}+1\right)+1+\xi^{2}}{2 \xi\left(e^{\alpha}+1\right)}=\frac{-\frac{e^{f(\xi)}}{\xi^{2}}+e^{f(\lambda)}}{\frac{2}{\xi} e^{f(\lambda)}\left(e^{\alpha}+1\right)} .
$$

Since the denominator in the last fraction in equation (3.28) is positive, we can show that $F<0$ if the numerator is negative. As $e^{f(\xi)} / \xi^{2} \rightarrow \infty$ for $\xi \rightarrow \infty$, we see that this is indeed the case for $\xi$ sufficiently large and fixed $\lambda$.

It follows from lemma 7 that there will in general be an even number (possibly zero) critical points of $\psi$ at $\eta=\pi$. The innermost will be a centre since $\partial F / \partial \xi>0$ here, the next one will be a saddle, and so on.

We now address the existence of critical points in the limits of small and large $\lambda$. We consider first the limit of small $\lambda$. Then the helix is an almost straight line and therefore no critical points are expected.

Lemma 8 There are no zeros of $F(\xi, \lambda)$ for small $\lambda$.

Proof First we consider the region $\xi<\lambda$ and we aim for an upper bound on $F(\xi, \lambda)$.

The second term in $F(\xi, \lambda)$ is disregarded

$$
F(\xi, \lambda) \leq \frac{1}{2} \xi-\frac{1}{2}\left(1+\lambda^{2}\right)^{\frac{1}{4}}\left(1+\xi^{2}\right)^{\frac{1}{4}} \frac{\sqrt{1+\xi^{2}}}{\xi} e^{f(\xi)} \frac{e^{-f(\lambda)}}{1+e^{f(\xi)-f(\lambda)}} .
$$

Using $1+e^{\alpha}<2, \xi>\lambda,\left(1+\lambda^{2}\right)^{\frac{1}{4}}\left(1+\xi^{2}\right)^{\frac{1}{4}}>1$ and $\frac{\sqrt{1+x^{2}}}{x} e^{f(x)}$ is increasing for all positive $x$ and $\lim _{x \rightarrow 0} \frac{\sqrt{1+x^{2}}}{x} e^{f(x)}=\frac{e}{2}$ so $\frac{\sqrt{1+\xi^{2}}}{\xi} e^{f(\xi)}>\frac{e}{2}$ we get from inequality (3.29)

$$
F(\xi, \lambda) \leq \frac{1}{2} \lambda-\frac{e^{1-f(\lambda)}}{8}
$$


Since $e^{f(x)}$ goes to zero for $x$ going to zero then $F(\xi, \lambda)<0$ for $\xi<\lambda$ and $\lambda$ small.

For $\xi>\lambda$ we return to the estimate (3.28). There exists a $c>0$ such that the function $e^{f(\xi)} / \xi^{2}>c$ for all $\xi>0$. Since $e^{f(\lambda)} \rightarrow 0$ for $\lambda \rightarrow 0$ it follows that $F<0$ for $\lambda$ sufficiently small.

We now turn to the case of large $\lambda$. Then the helix is densely wound and is close to being a cylindrical vortex sheet.

Lemma 9 There is at least two zeros of $F(\xi, \lambda)$ for large $\lambda$. One zero is located inside the helical cylinder and corresponds to a center, and one is located outside the helical cylinder and corresponds to a saddle.

Proof Since $F$ is continuous we can evaluate it at the vortex cylinder using the lower part of equation (3.21),

$$
F(\lambda, \lambda)=-\frac{1}{2 \lambda}-\frac{\lambda}{4 \sqrt{1+\lambda^{2}}} \ln (2)+\frac{1}{4}\left(\frac{1+\lambda^{2}}{\lambda}\right)
$$

which is positive for large $\lambda$. Since $F(\xi, \lambda)$ is negative for small and large $\xi$ an elliptic critical point in the inner region and a hyperbolic critical point in the outer region must exist for large $\lambda$.

We now complement our analytical results by a numerical investigation. The main results are shown in figure 3 . In agreement with the results above, no critical points exist for small values of $\lambda$. However, at $\lambda=1.265$ a saddle-node bifurcation occurs and a centre 
and a saddle are created in the outer region. At $\lambda=1.313$ the elliptic fixed point moves into the inner region. Increasing $\lambda$ yields no further bifurcations. While the location of a critical inside or outside the helix cylinder is not strictly speaking a topological property, it seems important physically to keep track of this. With this understanding, we conclude that three different flow topologies may occur in this flow.

The streamline patterns have reflection symmetry around the $x$-axis. This arises from the invariance of $\eta \rightarrow-\eta$ in equation (3.13) (and also in the original expression given by equation (3.3)). This symmetry is due to special properties of the field induced by a helical vortex filament and is not a general feature of helical flow fields.

\section{Topological effects of self-induced velocity}

The self-induced velocity on a vortex filament has been considered in e.g. [10, 33, 34, 4, 27]. Using the localised induction approximation (LIA) the self induced velocity on a helix with nonzero core size carrying vorticity is proportional to the binormal of the helix [27]:

$$
\boldsymbol{v}_{\mathrm{hel}}=\frac{\kappa \Gamma}{4 \pi} \ln \left(\frac{1}{\epsilon}\right) \boldsymbol{b}_{\mathrm{hel}},
$$

where $\epsilon$ is a non-dimensional core radius defined by the physical core radius $\sigma$ and the curvature $\kappa$,

$$
\epsilon=\sigma \kappa
$$

This means $0<\epsilon \leq 1$. Notice that $\boldsymbol{v}_{\text {hel }}$ is not defined for the core radius being zero. Equation (4.1) is valid up to order $\mathcal{O}(1)$ terms in $\epsilon$. Introducing

$$
\gamma=\frac{1}{4} \ln \left(\frac{1}{\epsilon}\right)
$$


(a)
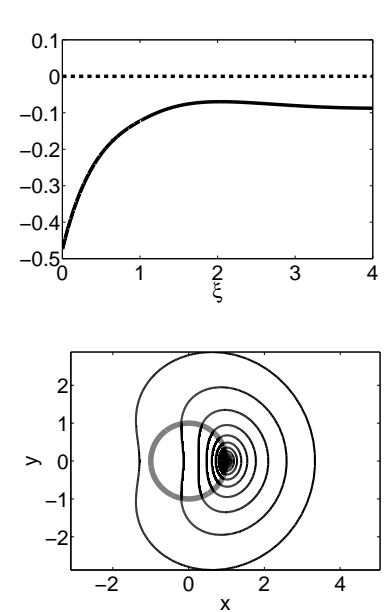

(b)
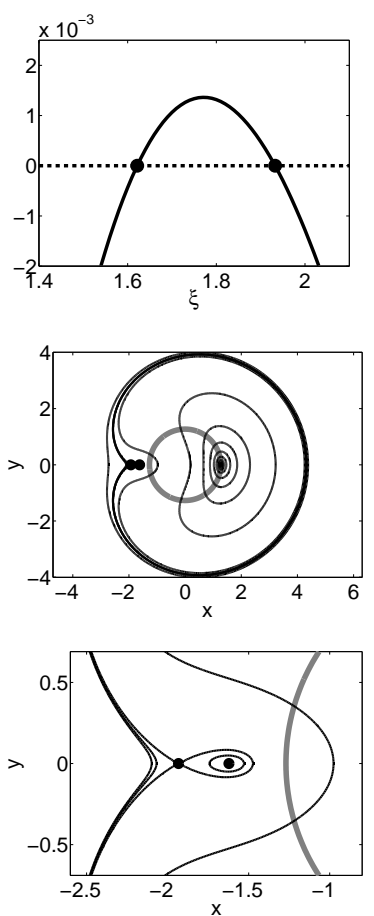

(c)
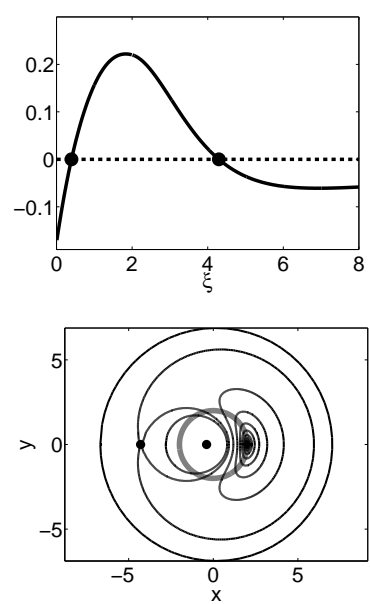

Figure 3. The three observed flow topologies of the closed form stream function $\psi_{H, c}$ equation (3.13). The top row shows graphs of $F(\xi, \lambda)$. The middle row shows isolines of the stream function $\psi_{H, c}$. Note the different scaling of the axes. The grey circle has radius $\lambda$ and represents the filament cylinder. (a) $\lambda=1.00$. No critical points of the stream function. (b) $\lambda=1.27$. Two critical points in the outer region. The panel in the third row is a detail near the critical points. (c) $\lambda=2.00$. The elliptic critical point is now located in the inner region.

the velocity of the helix may be written

$$
\boldsymbol{v}_{\text {hel }}=\kappa \frac{\Gamma}{\pi} \gamma \boldsymbol{b}_{\text {hel }}
$$

Therefore, increasing $\gamma$ increases the speed of the helix. By equation (4.3) an increase in $\gamma$ corresponds to a decrease in $\epsilon$ and thereby improves the accuracy of LIA.

By equation (4.4) the velocity of the vortex filament has constant components along 
the $\boldsymbol{e}_{z}$ and $\boldsymbol{e}_{\theta}$ directions (the component in the $\boldsymbol{e}_{r}$ direction is zero). Thus, any point on the helix is translated in the $z$-direction with the same speed, and rotated around the $z$-axis with same (negative) rotation frequency. Therefore, including finite core thickness in this way, the helix remains a helix as time varies. This hinges on $\boldsymbol{b}$ being a helical vector field.

\subsection{Topology of velocity field generated by constantly rotating and translating helical vortex filament}

Now we consider an thin helical vortex filament that translates along the center axis with constant speed and rotates around the center axis with constant speed. The case emerges from the selfinduced velocity on the helix for nonzero core size. However, the assumption is that the core size only affects the velocity of the filament itself and through that the entire flow. The ratio of rotation speed and translation speed is fixed as a consequence of LIA so the velocity is given by one parameter.

Introducing a non-negative parameter $\delta$ by

$$
\delta=\gamma \frac{1}{\sqrt{1+\lambda^{2}}}
$$

the stream function considered by Mezic et al. [27] (subscript $M$ ) can be written

$$
\psi_{M}(\xi, \eta)=\psi_{H}(\xi, \eta)-\frac{1}{2} \delta \xi^{2}
$$




\subsection{Closed form approximation of $\psi_{M}$}

Since the same products of modified Bessel functions appear in the expressions of Hardin and Mezic it is easy to construct a closed form approximation (subscript $c$ ) $\psi_{M, c}$ from equation (4.6),

$$
\psi_{M, c}=\psi_{H, c}-\frac{1}{2} \delta \xi^{2}
$$

with $\psi_{H, c}$ is given by equation (3.13). Many of the properties of $\psi_{H, c}$ are also valid for $\psi_{M, c}$ and are shown in the same way.

Since $\frac{\partial \psi_{M, c}}{\partial \eta}=\frac{\partial \psi_{H, c}}{\partial \eta}, \eta=0$ and $\eta=\pi$ are the only possible values of $\eta$ where critical points can occur. All information about critical points is contained in the function

$$
G(\xi, \eta, \lambda, \delta) \equiv \frac{\partial}{\partial \xi} \psi_{M, c}(\xi, \eta)=\frac{\partial}{\partial \xi} \psi_{H, c}(\xi, \eta)-\delta \xi
$$

with $\eta=0, \pi$. The zeroes of $G$ as a function of $\xi$ correspond to critical points, and the sign of the slope $\frac{\partial G}{\partial \xi}$ determines whether it is a center or a saddle.

We summarise the analytical results about the zeroes of $G$ in the following theorem. We omit the proof.

Theorem 2 (1) There are no zeroes of $G$ when $\lambda$ is small and $\delta$ is fixed for $\eta=0, \pi$.

(2) There are at least two critical points of $G(\xi, 0, \delta, \lambda)$ for large $\delta$ and $\lambda$ fixed.

(3) Zeroes for $G$ at $\eta=0$ can exist only when $\delta>\frac{1}{2}$.

(4) Zeroes for $G$ at $\eta=\pi$ can exist only when $\delta<\frac{1}{2}$.

(5) There are no zeroes for $G$ in the outer region $\xi<\lambda$ for $\eta=0$. 
(6) The innermost critical point, if it exists, is elliptic, the next is hyperbolic, and so on. This holds separately at $\eta=0, \pi$.

A main difference from the case considered in $\S 3.2$ is that critical points can also occur at $\eta=0$. However, they never exist simultaneously with critical points at $\eta=\pi$. This is a strict mathematical statement for $\psi_{M, c}$ whereas Mezic et al. [27] argue the same holds for $\psi_{M}$ invoking a numerical argument.

We now turn to a numerical investigation of $\psi_{M, c}$. Two new topologies are found. Figure 4(a) shows that for positive $\delta$ the hyperbolic critical point may be located in the inner domain. A critical point is possible for $\eta=0$ as shown in figure 4(b). Here the elliptic critical point is still closest to the origin but now the hyperbolic critical point is between the elliptic critical point and $(\lambda, 0)$. Only two critical points are observed at a given set of of parameters and the only bifurcations occurring are saddle-node bifurcations.

The reflection symmetry around the $x$ - axis is inherited from the invariance of $\eta \rightarrow-\eta$ in the stream function of Hardin and also the closed form formulation.

The critical point pattern as a function of the parameters is summarised in a bifurcation diagram, figure 5 . Here the parameter $\gamma=\sqrt{1+\lambda^{2}} \delta$ is used to facilitate comparison with the results of Mezic et al. [27] - the results compare very well. Three curves $k, l, m$ split the parameter space into regions with distinct topologies.

For small $\delta$ the topologies observed in the model of Hardin shown in figure 3 are recovered. The curves $l$ and $m$ intersect at $\gamma=0.07$ leaving only a very small region with two critical points in the outer region at $\eta=\pi$. The curve $k$ has a minimum at $\gamma=2.15$ which is then the threshold for critical points at $\eta=0$ to be present. The 
(a)
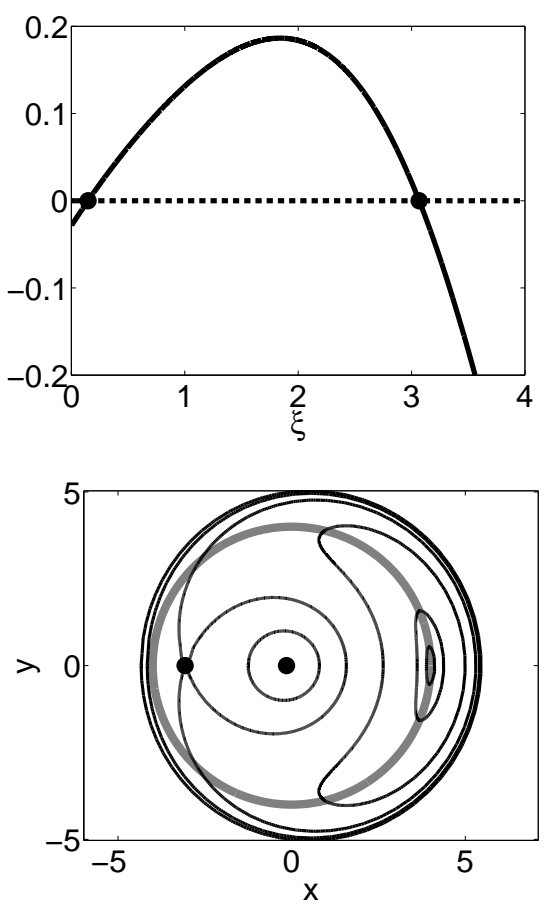

(b)
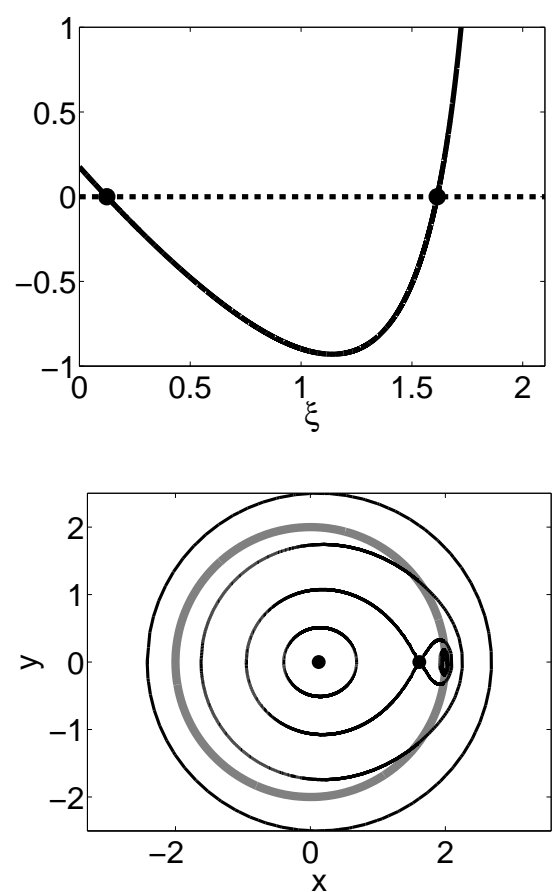

Figure 4. The two topologies of $\psi_{M, c}$ not existing for $\psi_{H, c}$. (a) $\lambda=4, \delta=0.3$. The top panel shows $G(\xi, \pi, 4,0.3)$. The bottom panel shows the contour plot of $\psi_{M, c}$. There are two critical points in the inner region $\xi<\lambda$. (b) $\lambda=2, \delta=2$. Top panel shows $G(\xi, 0,2,2)$. There are two critical points in the inner region but here the saddle point is closest to $(\lambda, 0)$.

curves $k$ and $l$ never cross but have a common asymptote which must be the line $\delta=\frac{1}{2}$, i.e. $\gamma=\frac{1}{2} \sqrt{1+\lambda^{2}}$ where no critical points can exist according to Theorem 2 . The curve $\gamma=\frac{1}{2} \sqrt{1+\lambda^{2}}$ approaches $\gamma=\frac{1}{2} \lambda$ for large $\lambda$ and this fits well with the figure.

Increasing $\gamma$ improves the accuracy of LIA as discussed in section 4 . In figure 6 the bifurcation diagram in figure 5 is superimposed with horizontal lines corresponding to various values of $\epsilon$ in equation (4.3). Decreasing $\epsilon$ corresponds to increasing the accuracy of LIA. The velocity field generated by the zero thickness helical vortex filament generates infinite speed of the vortex filament which is unphysical (as well as a zero thickness core). 


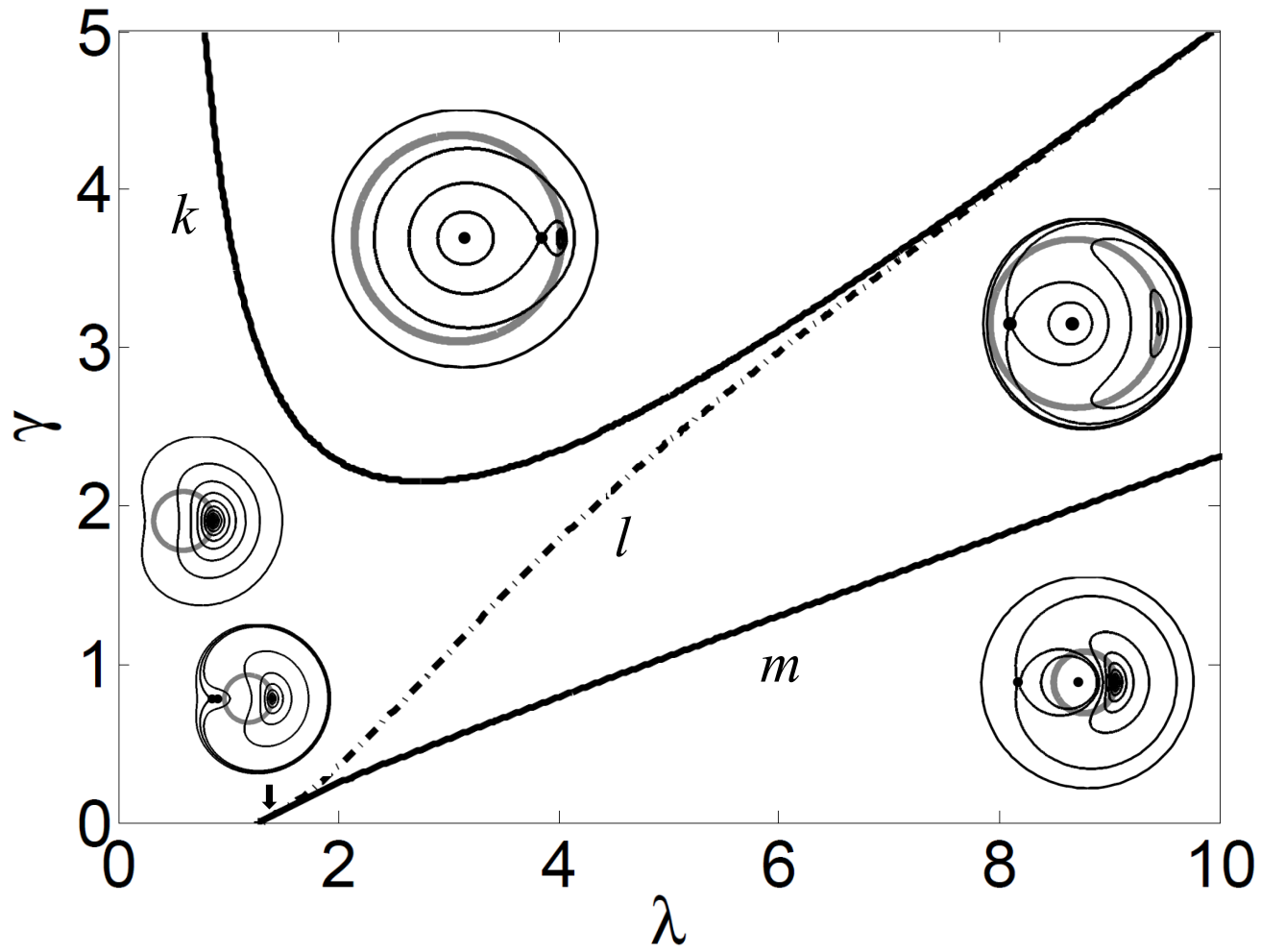

Figure 5. Bifurcation diagram for $\psi_{M, c}$. The top full curve $k$ is saddle-node bifurcation of critical points at $\eta=0$. The curve approaches the $\gamma$ axis asymptotically as shown in Theorem 2 (1) and (2). The dashed curve $l$ is saddle-node bifurcation of critical points at $\eta=\pi$. The lower full curve $m$ marks where the hyperbolic critical point at $\eta=\pi$ moves from the inner to the outer region. At $\gamma=0.07, l$ and $m$ intersect. Below that value they interchange their role with crossing $l$ corresponding to the elliptic critical point moving between the inner and outer region. Between the upper full curve and the dashed curve is the curve (not shown) $\delta=\frac{1}{2}$ i.e. $\gamma=\frac{1}{2} \sqrt{1+\lambda^{2}}$ where no critical points can occur.

However, Hardin's solution is useful for the velocity field away from the vortex filament. Using LIA the velocity field is no longer infinite on the filament. By demanding a high accuracy of LIA the topology with two critical points in the outer domain is not possible. 


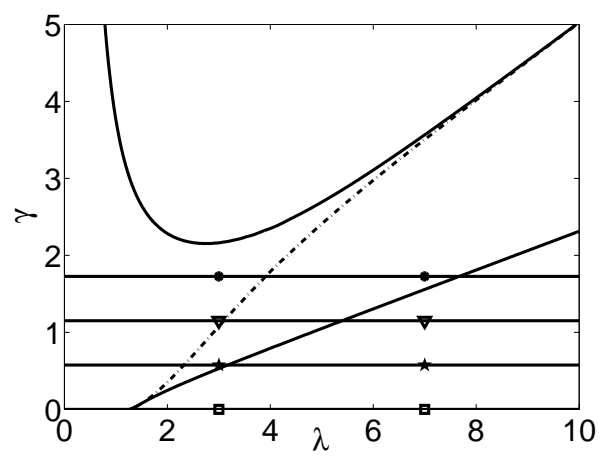

Figure 6. The bifurcation diagram from figure 5 with horizontal lines showing how well LIA works for the corresponding filament speed - see section 4. Circles: $\epsilon=10^{-3}$, triangles : $\epsilon=10^{-2}$, stars: $\epsilon=10^{-1}$, squares : $\epsilon=1$. Demanding $\epsilon \leq 10^{-3}$ only the part of the bifurcation diagram above the upper horizontal line is valid. Then the topology with two outer critical points is not accessible.

\section{Conclusions}

In theoretical mechanics coordinate-free formulations are generally very useful for highlighting fundamental consequences of symmetries. This point of view, however, seems to have had a quite limited impact on the research in helical fluid flows. We have extended the coordinate-free approach by Ettinger \& Titi [15] and shown how it clarifies the basic properties of these flows.

A main purpose of the present paper was a topological analysis of the streamlines in flows induced by helical vortex filaments. Using the closed-form approximation by Okulov [28] and Fukumoto \& Okulov [16] a significant simplification of the stream function was obtained, making a very complete analytical study of the flow topology possible. We have shown that a relatively small set of flow topologies can occur, even if self-induction of the filament is taken into account. Nevertheless, compared to the flow topology induced 
by rectilinear vortex filaments helical flow is much richer, underlining how complex these flows are.

There are obvious extensions of the topological analysis of the present paper, e.g. the flow induced by several helical vortex filaments $[29,30]$ and the flow in a domain bounded by a cylinder [20]. Work along these lines is in progress. Also, the topological analysis is not limited to inviscid flows. The same approach has previously been used to classify flow topologies in vortex breakdown in viscous axisymmetric flows, using both numerical computations $[8,7]$ and experimental data [25]. This could easily be extended to flows with helical symmetry.

\section{Acknowledgements}

We thank Valery Okulov for pointing out the closed-form approximation and for many fruitful discussions.

\section{References}

[1] M. Abramowitz and I. A. Stegun. Handbook of Mathematical Functions. Dep. of Commerce, USA, 1964.

[2] S. V. Alekseenko, P. A. Okulov, and S. I. Shtork. Helical vortices in swirl flow. J. Fluid Mech., 382:195-243, 1999.

[3] S.V. Alekseenko, P.A. Kuibin, and V.L. Okulov. Theory of concentrated vortices. SpringerVerlag Berlin Heidelberg, 2007.

[4] G.K. Batchelor. An introduction to fluids dynamics. Cambridge University Press, 1967.

[5] M. Brøns. Topological fluid dynamics of interfacial flows. Phys. Fluids, 6(8):2730-2737, 1994. 
[6] M. Brøns. Streamline topology: Patterns in fluid flows and their bifurcations. Adv. Appl. Mech., 41:1-42, 2007.

[7] M. Brøns and A. V. Bisgaard. Bifurcation of vortex breakdown patterns in a circular cylinder with two rotating covers. J. Fluid Mech., 568:329-349, 2006.

[8] M. Brøns, L. K. Voigt, and J. N. Sørensen. Streamline topology of steady axisymmetric vortex breakdown in a cylinder with co- and counter-rotating end-covers. J. Fluid Mech., 401:275-292, 1999.

[9] S. Childress, M. Landman, and H. Strauss. Steady motion with helical symmetry at large Reynolds number. In by H. K. Moffatt and A. Tsinober, editors, Topological fluid mechanics, proceedings of the IUTAM Symposium, Cambridge University Press. Cambridge University Press, 1990.

[10] L. S. Da Rios. Sul moto d'un liquido indefinito con un filetto vorticoso di forma qualunque. Rendiconti del Circolo Matematico di Palermo, 22:117-135, 1906.

[11] W. R. Dean. Note on the notion of fluid in a curved pipe. Phil. Mag., 4:208-223, 1927.

[12] I. Delbende, M. Rossi, and O. Daube. DNS of flows with helical symmetry. Theor. Comput. Fluid Dyn., 26:141-160, 2012.

[13] A. Deliceoğlu. Topology of two-dimensional flow associated with degenerate dividing streamline on a free surface. Euro. J. Appl. Math., 24(1):77-101, 2013.

[14] D. G. Dritschel. Generalised helical Beltrami flows in hydrodynamics and magnetohydrodynamics. J. Fluid Mech., 222:525-541, 1991.

[15] B. Ettinger and E. S. Titi. Global existence and uniqueness of weak solutions of threedimensional Euler equations with helical symmetry in the absence of vorticity stretching. SIAM J. Math. Anal., 41:269-296, 2009.

[16] Y. Fukumoto and V. L. Okulov. The velocity field induced by a helical vortex tube. Phys. Fluids, 17, 2005.

[17] M. Germano. The Dean equations extended to a helical pipe flow. J.Fluid Mech., 203:289305,2009 . 
[18] B.P. Gupta and R.G. Loewy. Theoretical analysis of the aerodynamic stability of multiple, interdigitated helical vortices. AIAA Journal, 12:1381-1387, 1974.

[19] J. C. Hardin. The velocity field induced by a helical vortex filament. Phys. Fluids, 25:19491952, 1982.

[20] R. H. A. Ijzermans, R. Hajmeijer, and P. J. van Langen. Accumulation of heavy particles around a helical vortex filament. Phys. Fluids, 19:107102, 2007.

[21] H. Ito. Flow in curved pipes. Jpn Soc. Mech. Engineers, 30:543-552, 2007.

[22] M. J. Landman. On the generation of helical waves in circular pipe flow. Phys. Fluids A, 2:738-747, 1990 .

[23] M. J. Landman. Time-dependent helical waves in rotating pipe flow. J. Fluid Mech., 221:289-310, 1990.

[24] S. Liu and J. H. Masliiyah. Axially invariant laminar flow in helical pipes with a finite pitch. J. Fluid Mech., 251:315-353, 1993.

[25] D. Lo Jacono, M. Nazarinia, and M. Brøns. Experimental vortex breakdown topology in a cylinder with a free surface. Phys. Fluids, 11:111704, 2009.

[26] D. Lucas and D. G. Dritschel. A family of helically symmetric vortex equilibria. J. Fluid Mech., 634:245-268, 2009.

[27] I. Mezic, A. Leonard, and S. Wiggins. Regular and chaotic particle motion near a helical vortex filament. Physica D, 111:179-201, 1998.

[28] V. L. Okulov. The velocity field induced by helical vortex filaments with cylindrical or conic supporting surface. Russ. J. Eng. Thermophys., 5:63-75, 1995.

[29] V. L. Okulov. On the stability of multiple helical vortices. J. Fluid Mech., 521:319-342, 2004 .

[30] V. L. Okulov and J. N. Sørensen. Stability of helical tip vortices in a rotor far wake. J. Fluid Mech., 576:1-25, 2007.

[31] F.W.J. Olver. The asymptotic expansion of Bessel functions of large order. Philos. Trans. R. Soc., A, 247:328-368, 1954. 
[32] F.W.J. Olver. The asymptotic solution of linear differential equations of the second order for large values of a parameter. Philos. Trans. R. Soc., A, 247:307-327, 1954.

[33] R. L. Ricca. The effect of torsion on the motion of a helical vortex filament. J. Fluid Mech., 273:241-259, 1994.

[34] R. L. Ricca. The contributions of Da Rios and Levi-Civita to asymptotic potential theory and vortex filaments dynamics. Fluid Dyn. Research, 18:245-268, 1996.

[35] L. G. Sarasua, A. C. Sicardi Schifino, and R. Gonzalez. The stability of steady, helical vortex filaments in a tube. Phys. Fluids, 11:1096-1103, 1998.

[36] J. N. Sørensen. Instability of helical tip vortices in rotor far wakes. J. Fluid Mech., 682:1-4, 2011.

[37] G.N. Throumoulopoulos and H. Tasso. Ideal magnetohydrodynamics equilibria with helical symmetry and incompressible flows. J. Plasma Physics, 62:449-459, 1999.

[38] E.R. Tuttle. Laminar flow in twisted pipes. J. Fluid Mech., 219:545-570, 1990.

[39] L. Zabielski and A.J. Mestel. Steady flow in a helically symmetric pipe. J. Fluid Mech., 370:297-320, 1998.

[40] L. Zabielski and A.J. Mestel. Unsteady flow in a helically symmetric pipe. J. Fluid Mech., 370:321-345, 1998 . 